\title{
Three-dimensional disk-based optical correlator
}

\author{
Kevin Curtis* \\ Demetri Psaltis, MEMBER SPIE \\ California Institute of Technology \\ Department of Electrical Engineering \\ Pasadena, California 91125 \\ E-mail: curtis@clockwise.att.com
}

\begin{abstract}
A total of 300 templates were stored as angularly multiplexed transmission holograms at multiple spots on a photopolymer disk. A novel correlator architecture is described and demonstrated that correlates all the templates against an input image within one disk rotation $(\approx 20 \mathrm{~ms})$. The projected performance of the system is 150,000 templates per disk and a processing rate of approximately 6000 correlations per second.
\end{abstract}

Subject terms: correlator; holographic correlator; photopolymer; holographic memories; optical processor.

Optical Engineering 33(12), 4051-4054 (December 1994).

\section{Introduction}

In recent years we have seen the implementation of several pattern recognition systems that use arrays of optical correlators to realize a variety of algorithms (e.g., template matching, radial basis functions, and neural networks). An array of correlators can be implemented by spatially multiplexing different filters on a two-dimensional (2-D) medium, frequently in a disk configuration. ${ }^{1-4}$ Alternatively, 3-D holographic storage can be used by superimposing multiple holograms utilizing either wavelength ${ }^{5}$ or angular ${ }^{6,7}$ multiplexing to form the correlation between the input and any stored reference. Finally, spectral and time domains can be used to store multiple filters in 2-D spectral hole-burning media. ${ }^{8}$ In this paper we present a method that combines angularly multiplexed storage in 3-D media with spatial multiplexing to form a 3-D disk. ${ }^{9}$ The 3-D disk allows us to store a very large number of gray-scale templates, whereas the disk rotation proves convenient for implementing the necessary shift in one of the two dimensions for the calculation of the image correlation function. The architecture we describe can be implemented with any holographic storage medium that can be fabricated into a thick slab (to allow volume holography) with sufficient area (to make a sizable disk). For instance, photorefractive crystals can be used. ${ }^{9}$ The 3-D disk used in the experimental demonstration in this paper is constructed out of HRF-150 DuPont photopolymer. ${ }^{10}$ In a previous publication $^{11}$ we have reported the storage of 10 angularly multiplexed holograms in this material.

*Current affiliation: AT\&T Bell Laboratories, 600 Mountain Avenue, Murray Hill, NJ 07974.

Paper 39034 received Mar. 24, 1994; revised manuscript received June 18, 1994 accepted for publication June 20, 1994

(C) 1994 Society of Photo-Optical Instrumentation Engineers. 0091-3286/94/\$6.00.

\section{Theory of Operation}

When a thick hologram is used to store the Fourier-transform filter in a VanderLugt correlator, the shift invariance is destroyed in one of the two dimensions. ${ }^{12}$ As a result, the output of such a system is simply one line of the 2-D correlation function. We can use angularly multiplexed holograms in a volume to store multiple templates and use the second dimension of the output plane to display in parallel one line of each of the 2-D correlation patterns between the input and the stored templates. By translating the input image, the 2-D correlation patterns are generated sequentially, one line at a time. ${ }^{2,6,7}$ In this paper we describe an alternative solution in which we translate the holographic medium instead. The reference holograms are stored by Fourier transforming in one direction and imaging in the other. Since the input is imaged onto the hologram in one dimension, translating the hologram produces the desired relative shift between input and reference that is necessary to compute the correlation function in two dimensions. Since the holographic medium is a 3-D disk, this translation is provided by the disk rotation. Moreover, in this architecture, we increase the number of templates by storing multiple angularly multiplexed holograms at multiple locations on the disk. Disk rotation is also conveniently used to access the different locations in sequence.

The optical setup is shown in Fig. 1. The image $f$ to be stored is presented to the system on a spatial light modulator (SLM-an Epson LCTV in the experiment). The first lens takes the Fourier transform of the image presented on the SLM. At the Fourier plane of the first lens, the image is filtered to edge-enhance it and also to remove the diffraction orders of the SLM. The second lens transforms this filtered signal back into an image that is demagnified by $1 / 5$. Using three cylindrical lenses, the image is imaged in the along-" track" $(x)$ direction and Fourier transformed in the radial (y) direction. The two spherical lenses, following the cylindrical 
lenses, demagnify the 1-D Fourier transform and 1-D image by a factor of $1 / 4$.

This pattern is then interfered with a plane wave with spatial frequency $\mu$ and stored in the thick photopolymer film laminated on a blank glass optical disk. Thus the pattern $f$ imaged in the $x$ direction and Fourier transformed in $y$ is holographically stored on the disk. Additional holograms are multiplexed at the same disk location by changing the angle of the reference beam using a rotating mirror. A $4 \mathrm{~F}$ system of lenses is used in the reference arm, as shown in Fig. 1, to change the incident angle of the reference beam without changing its location on the disk. The effective transmittance of the hologram can be written as $T(x, y) \approx \tilde{f} *(x, y / \lambda F)$ $\exp (j 2 \pi \mu x)$, where $\tilde{f}$ is the 1-D Fourier transform of $f$.

To generate the correlation function between a recorded function $f$ and an input function $g$, we present $g$ on the SLM. The function $g$ is mapped into the pattern $\tilde{g}(x, y / \lambda F)$ after going through the optical system that transfers it to the plane of the disk. The signal after the disk is given by $\tilde{g}(x, y / \lambda F) \tilde{f}^{*}(x, y / \lambda F) \exp (j 2 \pi \mu x)$. The lens placed between the disk and the detector array in Fig. 1 takes the 2-D Fourier transform of this signal. The result on the detector plane is given by ${ }^{12}$

$$
\begin{aligned}
S\left(x_{d}, y_{d}\right) \approx & \int_{-\infty}^{\infty} \int_{-\infty}^{\infty} \tilde{g}\left(\frac{x, y}{\lambda F}\right) \tilde{f} *\left(\frac{x, y}{\lambda F}\right) \\
& \times \exp \left\{-j \frac{2 \pi}{\lambda F}\left[x\left(x_{d}-\mu \lambda F\right)+y y_{d}\right]\right\} \mathrm{d} x \mathrm{~d} y \\
& \times \operatorname{sinc}\left(\frac{L \mu}{F}\left(x_{d}-\mu \lambda F\right)\right),
\end{aligned}
$$

where $L$ is the thickness of the disk. The different plane-wave references ( $\mu$ 's) used to record each angularly multiplexed hologram result in the reconstructions being uniquely shifted on the detector plane to $x_{d}=\mu \lambda F$. In addition, the sinc function in Eq. (1) places a window in the $x_{d}$ direction, which has the benefit that it reduces the crosstalk between different correlations. Specifically, the line detectors are spaced in the $x_{d}$ direction by $\Delta x_{d}=n F / L \mu$, where $n$ is a small integer. This places each of the adjacent line detectors in the $n$ 'th nulls of its neighbors, thereby minimizing crosstalk. Writing the product of Fourier transforms in the $y$ direction as a correlation function and observing the light amplitude at $x_{d}=\mu \lambda F$, we obtain from Eq. (1)

$S\left(\mu \lambda F, y_{d}\right) \approx \int_{-\infty}^{\infty} \int_{-\infty}^{\infty} g(x, y) f\left(x, y-y_{d}\right) \mathrm{d} x \mathrm{~d} y$.

This is an inner product in the $x$ direction and correlation in $y$. In other words, it is one line of the full 2-D correlation function between $f$ and $g$.

Using disk rotation as approximately a linear shift $\theta(t)$ in the $x$ direction generates the full 2-D correlation function with time:

$S^{\prime}\left(\mu \lambda F, y_{d}, t\right) \approx \int_{-\infty}^{\infty} \int_{-\infty}^{\infty} g(x, y) f\left[x+\theta(t), y-y_{d}\right] \mathrm{d} x \mathrm{~d} y$.

The intensity at the detector is $I\left(\mu \lambda F, y_{d}, t\right)=S^{\prime} S^{\prime *}$, and therefore the detected signal is the square of the correlation

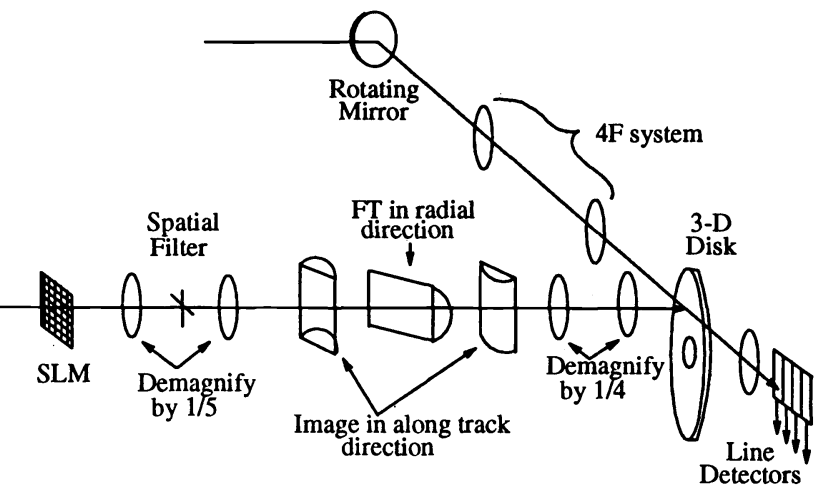

Fig. 1 3-D disk-based optical-correlator setup.

function. With a thick medium, multiple holograms can be recorded at a single spot on the disk, using angle multiplexing. Therefore, different 2-D correlation functions for each multiplexed hologram can be generated one line at a time and detected in parallel.

\section{Disk Rotation Effects}

In this architecture we make use of disk rotation during reconstruction to shift the stored images with respect to the input in one dimension $(x)$ and thus form the complete correlation. Disk rotation, however, is only approximately equivalent to a shift. In this section we analyze the effects due to rotation of the image. We analyze this effect ignoring the effects due to the finite thickness of the hologram, since the observed rotation effects are identical for 2-D and 3-D holograms. We calculate the effects of rotation by considering the correlation between an individual pixel at the input image and a pixel of one of the images stored in the hologram. The signal at the plane of the disk is focused in the $x$ direction and a plane wave in the $y$ direction, and therefore can be written as $S \approx \exp (-j 2 \pi \beta y) \operatorname{rect}(x / \delta)$, where $\beta$ is the spatial frequency in the $y$ direction and $\delta$ is the pixel size. This signal is stored with a plane-wave reference $R=\exp (-j 2 \pi \mu x)$ with spatial frequency $\mu$ in the $x$ direction. The recorded hologram can be written as

$R S^{*}=\exp (-j 2 \pi \mu x) \exp (j 2 \pi \beta y) \operatorname{rect}(x / \delta)$.

The hologram is then rotated by $\mathrm{d} \theta$ about a center of rotation in the $x$ - $y$ plane a distance $r$ away from the center of the $x, y$ coordinates. This rotation results in the coordinates being transformed according to $x^{\prime} \approx x-(y+r) \mathrm{d} \theta$ and $y^{\prime} \approx y+x \mathrm{~d} \theta$. Substituting these into Eq. (4), the hologram can be expressed in terms of the unrotated coordinates $(x, y)$ as

$$
\begin{aligned}
R S^{*}= & \exp \{j 2 \pi \mu[x-(y+r) \mathrm{d} \theta]\} \exp (j 2 \pi \beta y) \\
& \times \exp (j 2 \pi \beta \mathrm{d} \theta x) \operatorname{rect}\left[\frac{x-(y+r) \mathrm{d} \theta}{\delta}\right] .
\end{aligned}
$$

We multiply the above expression by $S$ to generate the 1-D inner product and 1-D correlation. After we expand and simplify the exponents, we obtain 


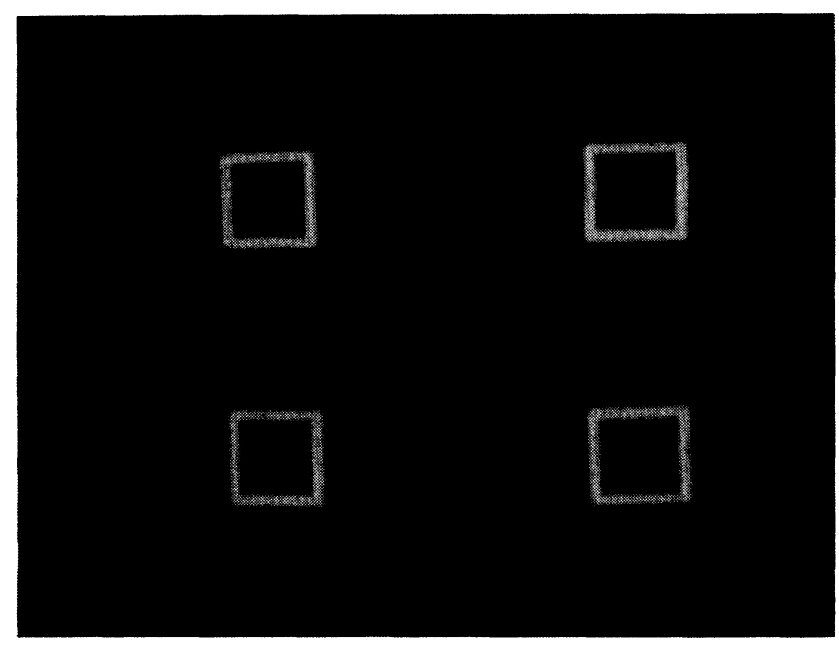

Fig. 2 Image used to test correlator.

$S R S^{*}=\exp (j 2 \pi \mu x) \exp (-j 2 \pi \mu \mathrm{d} \theta y)$

$\times \exp (j 2 \pi \beta \mathrm{d} \theta x) \operatorname{rect}\left[\frac{x-(y+r) \mathrm{d} \theta}{\delta}\right]$

$\times \operatorname{rect}\left(\frac{x}{\delta}\right)$.

After the Fourier-transforming lens, the terms in Eq. (6) correspond to the desired signal and shifts of this signal on the detector plane, depending on the amount of rotation $\mathrm{d} \theta$. The terms and their effects are listed below:

- $\exp (j 2 \pi \mu x)$ is the reconstructed plane wave without rotation-the desired signal.

- $\exp (-j 2 \pi \mu \mathrm{d} \theta y)$ corresponds to a shift in the $y$ direction on the detector plane, and it can be compensated by simply reinterpreting the detected signal.

- $\exp (j 2 \pi \beta \mathrm{d} \theta x)$ corresponds to a shift in the $x$ direction on the detector plane, and it can be neglected if $N^{2} \delta / r<1$, where $N$ is the number of pixels in one dimension.

- $\operatorname{rect}[(x-(y+r) \mathrm{d} \theta) / \delta] \operatorname{rect}(x / \delta)$ is due to the finite pixel size. The relative rotation between the two rect functions is negligible if $y<r / N$.

In our experimental setup, the shift of the correlation pattern was almost entirely in the $y$ direction. If the above conditions for neglecting the rotation effects are not satisfied, the result is a reduction in the shift invariance of the correlator in the $x$ direction.

\section{Experimental Correlation}

Using the system shown in Fig. 1, 300 transmission holograms were stored at a radius of $5 \mathrm{~cm}$ along a "ring" around the disk. Using DuPont's HRF-150 film and exposing with 488-nm light, three holograms were angularly multiplexed at each spot on the disk. The disk was then rotated to expose a total of 100 different spots; since at each spot three holograms were recorded, a total of 300 holograms were stored. The size of the illuminated region was approximately

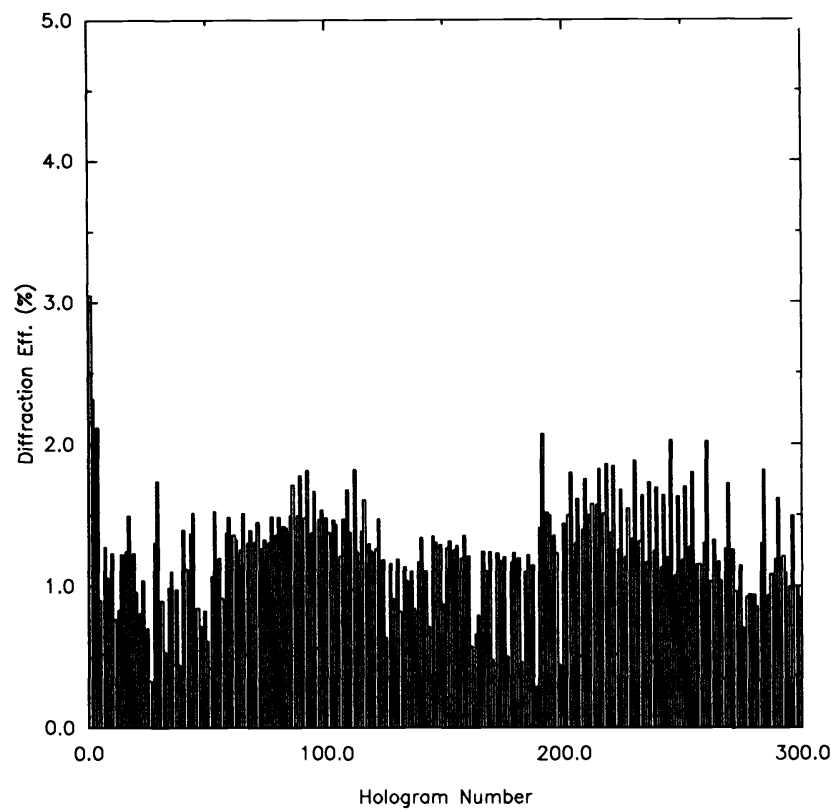

Fig. 3 Diffraction efficiency of 300 holograms-three holograms at a spot with 100 spots on a ring of a 3-D disk.

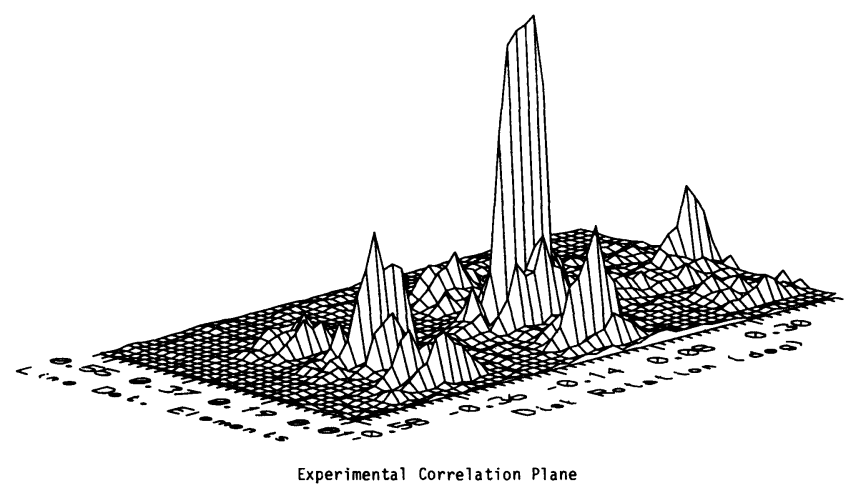

Fig. 4 Experimental autocorrelation squared of the pattern shown in Fig. 2 and produced using the optical disk-based correlator.

$0.9 \times 0.6 \mathrm{~mm}$ with a signal intensity of $2.4 \mathrm{~mW} / \mathrm{cm}^{2}$ and a reference-beam intensity of $2.0 \mathrm{~mW} / \mathrm{cm}^{2}$. After each spot was sensitized with preillumination ${ }^{11}$ using the reference beam for $25 \mathrm{~s}$, each hologram was exposed for $10 \mathrm{~s}$. The disk rotation between spots was $3.5 \mathrm{deg}$, and the angular separation of the three multiplexed holograms was $1.5 \mathrm{deg}$. The holograms were made with the image of four boxes shown in Fig. 2. The diffraction efficiency for the 300 holograms is shown in Fig. 3. The 3-D disk was made by pressing (laminating) the $38-\mu \mathrm{m}$-thick photopolymer film onto the glass disk.

Figure 4 is an experimental plot of the autocorrelation function squared of the four-box image in Fig. 2. It agrees reasonably well with the expected autocorrelation of this image. Figure 4 was generated by storing the image on the disk as explained above, and then presenting the same image as input to the system. The correlation plane was sampled by a linear CCD detector array. In this experiment the disk was not continuously rotated. Instead, the detector was read out, the disk was rotated by $0.02 \mathrm{deg}$, and the process repeated 


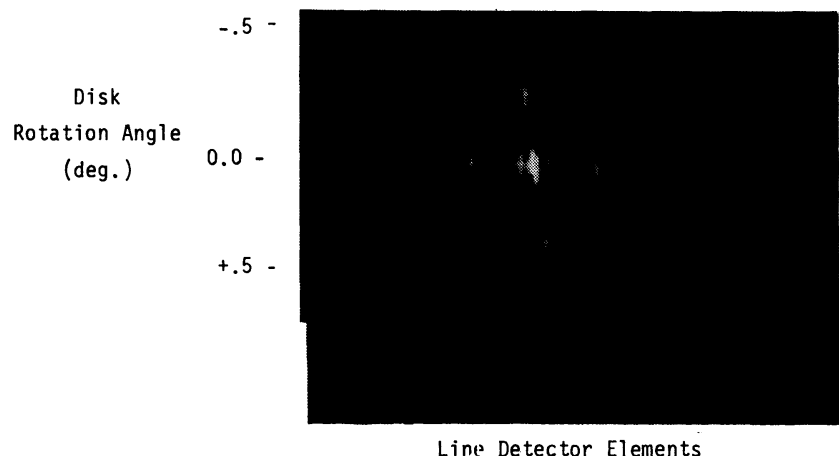

Fig. 5 2-D gray-scale depiction of the pattern in Fig. 4, showing the slant of the correlation pattern due to disk rotation.

60 times to obtain 60 lines of the 2-D correlation pattern. Since the boxes occupy a large portion of the input plane (SLM), Fig. 4 demonstrates that the correlation ability exists across most of the input plane. Figure 5 is the gray-scale image representation of the correlation function in Fig. 4. Notice the slant in the correlation pattern that is due to disk rotation. The shift produced by the experimental setup was mostly in the $y$ direction (labeled line detector elements in Fig. 5), as predicted.

\section{Discussion}

We have demonstrated a multichannel disk-based optical correlator using both angular and spatial multiplexing of volume holograms. The full 2-D correlation function of an input image against stored templates is generated by using disk rotation to shift one image over the other, thus reading out one line of each correlation function in parallel. A total of 300 holograms, in the required 1-D image and 1-D Fouriertransformed format, were stored on a single ring of an optical disk. The correlation generation was experimentally verified and compared with theory.

The thickness of the material was about $38 \mu \mathrm{m}$. Using the spin-coating technique, we have made a photopolymer film thick enough $(\approx 300 \mu \mathrm{m})$ to store more than 50 holograms in one spot. Using the whole area of the disk and leaving enough blank space between spots, approximately 3000 spots can be stored on a 5-cm-radius disk. Therefore, if polymer film of approximately $300-\mu \mathrm{m}$ thickness can be manufactured, then this results in a storage capacity of $\approx 150,000$ holograms or templates. The correlator described above can achieve very high correlation rates without the need of fast SLM. However, the processing bottleneck is shifted to the detectors. The speed of the current correlator is limited by the speed of the linear CCD detector arrays. A linear detector array is needed for each angular multiplexed hologram position. For example, if 50 holograms are multiplexed at each spot, then 50 linear detector arrays are employed, each with several hundred pixels. With the 50 holograms per spot and linear CCD detector arrays with 500 elements each running at $30 \mathrm{MHz}$, the correlation rate is about 6000 correlations per second. Less than $10 \mathrm{~mW}$ of light incident on the film is sufficient to realize this correlation rate, assuming a measured diffraction efficiency of $2 \times 10^{-3}$ when 50 holograms were multiplexed in a $38-\mu \mathrm{m}$-thick film. ${ }^{13}$ The correlation rate could be significantly increased using custom detector arrays that perform a winner-take-all operation on the pixels and report the location and magnitude of the largest correlation peak off chip. Note that the above performance is achievable without the need to develop new components.

\section{Acknowledgments}

This work was supported by AFOSR and Northrop. The photopolymer was graciously made available to use by DuPont; in particular, the authors wish to thank Jay Calio of DuPont. In addition, we appreciate the many helpful discussions with Dr. Sidney Li.

\section{References}

1. D. Psaltis, M. A. Neifeld, and A. Yamamura, "Image correlators using optical memory disks," Opt. Lett. 14(9), 429-431 (1989).

2. D. Psaltis, M. A. Neifeld, A. Yamamura, and S. Kobayashi, "Optical memory disks in optical information processing," Appl. Opt. 29(14), 2038-2057 (1990)

3. A. Kutanov, B. Abdrisaev, and S. Dordoev, "Holographic disked-based optical neural network,"' Opt. Lett. 17(13), 952-954 (1992).

4. E. Gulanyan, A. L. Mikaelyan, L. V. Molchanova, V. A. Sidorov, and I. V. Fedorov, "Use of a photothermoplastic disk in memories based on one-dimensional holograms,"' Sov. J. Quantum Electron. 19(8), 1124-1126 (1989)

5. F. T. S. Yu, S. Wu, A. W. Mayers, and S. Rajan, “Wavelength multiplexed reflection matched spatial filters using $\mathrm{LiNbO}_{3}$,' Opt. Comm 81(6), 343-347 (1991).

6. F. H. Mok, M. C. Tackett, and H. M. Stoll, "Massively parallel optical template matcher/correlator," presented at OSA Annual Meeting, Boston, 1990, Paper ThII4.

7. C. Gu, J. Hong, and S. Campbell, "2-D shift-invariant volume holographic correlator,' Opt. Commun. 88, 309-314 (1992).

8. X. A. Shen and R. Kachru, "High speed pattern recognition by using stimulated echoes," Opt. Lett. 17(9), 520-522 (1992).

9. D. Psaltis, "Parallel optical memories," Byte 17(9), 179-182 (1992).

10. W. K. Smothers, T. J. Trout, A. M. Weber, and D. J. Mickish, "Hologram recording in DuPont's new photopolymer material,", presented at 2nd Int. Conf. on Holographic Systems, Bath, U.K. (1989).

11. K. Curtis and D. Psaltis, "Recording of multiple holograms in photopolymer films," Appl. Opt. 31(35), 7425-7428 (1992).

12. J. Yu, F. Mok, and D. Psaltis, "Capacity of optical correlators," San Diego, CA, Proc. SPIE 825-22 (1987).

13. K. Curtis, A. Pu, and D. Psaltis, "A method for holographic storage using peristrophic multiplexing,' Opt. Lett. 19(13), 993-994 (1994).

Kevin Curtis received his BS, MS, and PhD degrees in electrical engineering in 1990,1992, and 1994, respectively, all from California Institute of Technology, Pasadena. Dr. Curtis has authored or coauthored over 20 publications on holography. He is a member of both OSA and SPIE, and his current research interests include holography, optical information processing, photopolymers, photorefractives, and optical memories. He is currently a postdoctoral member of the technical staff at AT\&T Bell Labs, Murray Hill, NJ.

Demetri Psaltis received the BSc in electrical engineering and economics in 1974 and the MSc and PhD degrees in electrical engineering in 1975 and 1977, respectively, all from Carnegie-Mellon University, Pittsburgh. He remained at Carnegie-Mellon, as a research associate and later as a visiting assistant professor, for three years. In 1980, he joined the faculty at the California Institute of Technology, Pasadena, where he is now executive officer of the Computation and Neural Systems Department, professor of electrical engineering, and a consultant to industry. His research interests are in optical memories, optical information processing, holography, pattern recognition, neural networks, and optical devices. Dr. Psaltis has authored or coauthored over 200 publications. He is a fellow of the Optical Society of America and received the International Commission on Optics Prize in 1989. 